\title{
Reducción del dolor postoperatorio con rangos de neumoperitoneo de bajo volumen en cirugía laparoscópica
}

\author{
Postoperative pain reduction with low-volume \\ pneumoperitoneum ranges in laparoscopic surgery \\ Adán Araujo-López, * Sergio Javier Herrera-Barrón, ${ }^{\ddagger}$ \\ Jesús Giovanni Insunza-Miranda, ${ }^{\ddagger}$ Ana Gabriela Sierra-Brozon ${ }^{\S}$
}

\begin{abstract}
RESUMEN. Introducción: En la actualidad, la colecistectomía laparoscópica se considera el procedimiento de elección para el manejo de la colelitiasis sintomática. Se ha propuesto que el neumoperitoneo en la cirugía laparoscópica incrementa el dolor postquirúrgico y produce efectos fisiológicos adversos en algunos pacientes. Objetivo: Controlar el dolor postoperatorio en cirugía, reduciendo la presión de neumoperitoneo. Material y métodos: Estudiar en tres hospitales (dos públicos y uno privado) para comparar la técnica convencional mediante la presión intraabdominal original con bajar la presión del neumoperitoneo hasta $10 \mathrm{mmHg}$ para reducir el dolor. Resultados: Con base en el estudio en el que se comparó con la técnica convencional con presión normal, mostramos una diferencia significativa (con una $\mathrm{p}=0.002$ ) para la reducción del dolor postoperatorio, reduciendo, en promedio, el neumoperitoneo con una presión transoperatoria de $10.9 \mathrm{mmHg}$ de $\mathrm{CO}_{2}$. Conclusión: Reducir la presión de $\mathrm{CO}_{2}$ en $\mathrm{mmHg}$ hasta 10.9 en promedio del neumoperitoneo transoperatorio mostró una presión segura y sin complicaciones, además de que redujo el dolor postoperatorio sin necesidad de escalar hacia un mayor número de analgésicos.
\end{abstract}

Palabras clave: Neumoperitoneo, dolor postoperatorio, cirugía laparoscópica.

ABSTRACT. Introduction: At present, laparoscopic cholecystectomy is considered the procedure of choice in the management of symptomatic cholelithiasis. It has been proposed that pneumoperitoneum in laparoscopic surgery increases post-surgical pain and produces adverse physiological effects in some patients. Objective: Control postoperative pain in surgery by reducing pneumoperitoneum pressure. Material and methods: Study 3 hospitals, both public and private, comparing the conventional technique using the original intraabdominal pressure versus lowering the pneumoperitoneum pressure to $10 \mathrm{mmHg}$, to reduce pain. Results: Based in the comparison against the conventional technique with normal pressure we described a significant difference $(p=0.002)$ for less postoperative pain with pneumoperitoneum technique with a mean decrease of

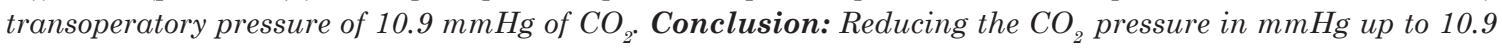
on average of the transoperative pneumoperitoneum, was shown as a safe, uncomplicated pressure and reduced postoperative pain without the need to scale up a greater number of analgesics.

Keywords: Neumoperitoneum, postsurgical pain, laparoscopic surgery.

* Hospital General de Querétaro. Santiago de Querétaro. Qro. México.

‡ Hospital de San Juan del Río. San Juan del Río, Qro. México.

$\S$ Hospital San José de Querétaro. Santiago de Querétaro, Qro. México.

Recibido: 13 de Noviembre de 2019.

Aceptado: 29 de Junio de 2020.

\section{Correspondencia:}

Adán Araujo-López

Circuito Andamaxei No. 6, Col. Paseos del Bosque, CP 76910, Corregidora, Querétaro. Teléfono: 442744 8305. Celular: 4444491203.

E-mail: draraujolopez@gmail.com

Conflicto de intereses: Los autores declaran no tener conflicto de intereses. 


\section{INTRODUCCIÓN}

En la actualidad, la colecistectomía laparoscópica se considera como el procedimiento de elección para el manejo de la colelitiasis sintomática. Se ha propuesto que el neumoperitoneo de la cirugía laparoscópica incrementa el dolor postquirúrgico y produce efectos fisiológicos adversos en algunos pacientes. ${ }^{1}$

Dado que la insuflación peritoneal disminuye el retorno venoso y reduce el gasto cardiaco, esto puede representar un peligro para pacientes con reserva cardiaca baja. ${ }^{2}$ Por tal razón, algunos estudios han mostrado ventajas potenciales con el uso rutinario de presiones bajas para mantener el neumoperitoneo durante la ejecución de la colecistectomía laparoscópica. ${ }^{2}$ Sin embargo, no existe un acuerdo general con respecto a qué presión es la que debe mantenerse en el neumoperitoneo. Al respecto, tres estudios prospectivos y aleatorios ya publicados muestran de manera significativa un menor dolor postquirúrgico y escapular, cuyas tasas de conversión y complicaciones son similares cuando la colecistectomía laparoscópica se practica con un neumoperitoneo de baja presión. A pesar de esta evidencia, algunos otros estudios más recientes no encuentran diferencias significativas en cuanto al dolor postquirúrgico entre pacientes sometidos a neumoperitoneo de baja presión y aquéllos sometidos a una presión convencional. ${ }^{1-9}$

Hace poco tiempo, la Asociación Europea de Cirugía Endoscópica publicó sus guías prácticas sobre el neumoperitoneo para la cirugía laparoscópica. En éstas, recomienda usar la menor presión intraabdominal posible que permita una adecuada exposición del campo quirúrgico; también recomiendan no utilizar una presión rutinaria (recomendación grado B). ${ }^{10}$

De acuerdo con estas guías, una presión intraabdominal inferior a $14 \mathrm{mmHg}$ se considera segura en un paciente sano (recomendación grado A). ${ }^{1-3}$

La omalgia es un evento adverso postoperatorio frecuente que se presenta entre 35 a $63 \%$ de los casos, suele ser de corta duración y de baja intensidad, con un pico de entre 24-48 horas tras la colecistectomía laparoscópica. ${ }^{4,11-16}$

La presión estándar que se utiliza es de 12-14 $\mathrm{mmHg}$. Para tratar de disminuir el impacto de los efectos adversos del neumoperitoneo (como reflejo vasovagal, arritmias cardiacas, acidosis e hipercapnia), minimizar el efecto hemodinámico y el dolor postoperatorio, se ha empleado un rango de baja presión (7-11 $\mathrm{mmHg}$ ) durante el neumoperitoneo. Sin embargo, las presiones más bajas podrían resultar en una exposición limitada, lo que provocaría aumento del tiempo quirúrgico, conversión a presión laparoscópica estándar o conversión a colecistectomía abierta. El objetivo de este estudio es comparar las ventajas del neumoperitoneo de bajo volumen frente al neumoperitoneo con presión estándar para el manejo de dolor postoperatorio. ${ }^{1-5}$

\section{MATERIAL Y MÉTODOS}

Estudio retrospectivo, descriptivo, observacional y comparativo. El trabajo incluyó pacientes intervenidos quirúrgicamente por diferentes cirujanos de tres hospitales: dos hospitales públicos en los cuales se utilizó la metodología original (Hospital General de Querétaro y Hospital Cadereyta) y un hospital del medio privado (Hospital San José de Querétaro). Los casos de los hospitales públicos conformaron el grupo control y los del hospital privado el grupo en reto (neumoperitoneo con presión baja de 9-11 $\mathrm{mmHg}$ ).

Las variables analizadas fueron: edad, sexo, comorbilidades (diabetes mellitus, hipertensión arterial y otros), presión intraabdominal (PIA) inicial y transquirúrgica, y complicaciones presentadas. El dolor postquirúrgico fue evaluado mediante una escala visual análoga (EVA).

Análisis estadístico. Se utilizó estadística descriptiva, prueba t de Student para variables cuantitativas y chi cuadrada $\left(\chi^{2}\right)$ para variables cualitativas. El análisis se realizó con el programa SPSS v.21. Los resultados se presentan en tablas.

Aspectos éticos: La presentación de este manuscrito cuenta con la aprobación de la oficina 


\begin{tabular}{|c|c|}
\hline Variable & $n(\%)$ \\
\hline \multicolumn{2}{|l|}{ Género } \\
\hline Hombre & $16(19.5)$ \\
\hline Mujer & $66(80.5)$ \\
\hline Edad & $39.7 \pm 15.2$ \\
\hline \multicolumn{2}{|l|}{ Comorbilidades } \\
\hline Sí & $22(26.8)$ \\
\hline No & 60 (73.2) \\
\hline DM & $14(17.1)$ \\
\hline HAS & $10(12.2)$ \\
\hline FA & $1(1.2)$ \\
\hline Urolitiasis & $1(1.2)$ \\
\hline Hepatopatía & $3(3.7)$ \\
\hline \multicolumn{2}{|l|}{ Diagnóstico } \\
\hline $\mathrm{CCL}$ & $57(69.5)$ \\
\hline $\mathrm{CCL}+$ piocolecisto & $2(2.4)$ \\
\hline $\mathrm{CCL}+$ hidrocolecisto & $11(13.4)$ \\
\hline Quiste hepático & $1(1.2)$ \\
\hline Absceso hepático & $1(1.2)$ \\
\hline $\mathrm{CCL}+$ coledocolitiasis & $10(12.2)$ \\
\hline \multicolumn{2}{|l|}{ Cirugía } \\
\hline Colecistectomía laparoscópica & $74(96.3)$ \\
\hline Drenaje laparoscópico & $2(2.4)$ \\
\hline $\begin{array}{l}\text { Colecistectomía laparoscópica + } \\
\text { colangiografía + exploración } \\
\text { de vías biliares }\end{array}$ & $1(1.2)$ \\
\hline PIA inicio $(\mathrm{mmHg})$ & $13.97 \pm 0.22$ \\
\hline PIA durante la cirugía $(\mathrm{mmHg})$ & $13.06 \pm 1.58$ \\
\hline PIA promedio (mmHg) & $12.86 \pm 0.43$ \\
\hline Dolor postquirúrgico & $5.8 \pm 2.11$ \\
\hline
\end{tabular}

de enseñanza e investigación de la institución y el consentimiento informado del paciente.

\section{RESULTADOS}

La muestra de este estudio fue de 82 pacientes. Hubo mayor número de mujeres intervenidas. Predominaron los casos sin comorbilidades asociadas y los diagnósticos de colecistitis crónica litiásica. El promedio de dolor, evaluado mediante EVA, fue de 5.8 (Tabla 1).
La Tabla 2 muestra los resultados obtenidos en uno y otro grupo. Al comparar la incidencia de comorbilidades del grupo control (n $=10,17.5 \%)$ frente a la del grupo con presión intraabdominal baja $(\mathrm{n}=12,48 \%)$ se registró diferencia significativa (0.006), lo que implica menor sesgo de falsos negativos. También se observó diferencia significativa en la presión intraabdominal empleada durante la cirugía $(\mathrm{p}=$ 0.001), así como en la presión intraabdominal media de cada grupo $(p=0.004)$.

La comparación del dolor postquirúrgico mostró diferencia estadística significativa ( $\mathrm{p}=$ 0.002) en favor del grupo neumoperitoneo con presión baja frente al grupo control (EVA: $4.7 \pm$ 2.1 versus $6.2 \pm 1.92)$.

Las comorbilidades que se registraron con mayor frecuencia fueron diabetes mellitus e hipertensión arterial sistólica en ambos grupos; no obstante, el porcentaje de incidencia fue mayor en el grupo neumoperitoneo con baja presión que en el grupo control, lo cual se traduce en que, incluso con neuropatía, el dolor se mostró en menor proporción.

La presión de insuflación inicial de la cavidad abdominal fue de $14 \mathrm{mmHg}$ en ambos grupos. El promedio de presión de $\mathrm{CO}_{2}$ transquirúrgico fue de $10.9 \mathrm{mmHg}$ en el grupo de neumoperitoneo con baja presión y $14 \mathrm{mmHg}$ en el grupo control $(p=0.004)$, lo cual indica que \pm $4 \mathrm{mmHg}$ sí fueron suficientes para demostrar una mejoría del dolor en el grupo manejado con menor presión intraabdominal $(\mathrm{p}=0.002)(T a-$ bla 2). Con base en esta diferencia, podemos decir que entre menor presión maneje el $\mathrm{CO}_{2} \mathrm{del}$ neumoperitoneo, mejor se puede manipular el dolor, independientemente de si se coloca un bloqueo neuromuscular o no. 
$5 \mathrm{~cm}$. Ésta ocupa la fosa de la vesícula biliar de la cara visceral del hígado a nivel del lóbulo derecho, en la unión del tercio medio con los dos tercios laterales del borde anterior. ${ }^{1}$ Tiene tres partes: el fondo, el cuerpo y el cuello. El fondo normalmente se localiza a nivel del noveno cartílago costal derecho y a nivel de la línea media clavicular. El cuerpo se encuentra en íntimo contacto con la segunda porción del duodeno y el colon. El cuello se dirige hacia el hilio hepático y continúa con el conducto cístico; éste se une con el conducto hepático común para formar el conducto del colédoco o conducto biliar común, que mide alrededor de $15 \mathrm{~cm}$ de largo. ${ }^{1}$

La vesícula no se encuentra enteramente rodeada por peritoneo, sólo el fondo tiene un revestimiento peritoneal completo, que describe por encima de un pequeño receso. El cuerpo está peritonizado en su cara inferior y en las laterales y, desde ahí, el peritoneo se refleja sobre la superficie del hígado. A nivel del cuello, el peritoneo forma un meso, que se inserta en la cara inferior del hígado y se denomina mesocisto. Éste a menudo se prolonga

\begin{tabular}{|c|c|c|c|}
\hline Variable & $\begin{array}{c}\text { Neumoperitoneo a } \\
\text { presión estándar }(\mathrm{n}=57) \\
\mathrm{n}(\%)\end{array}$ & $\begin{array}{c}\text { Neumoperitoneo a } \\
\text { 9-11 mmHg }(n=25) \\
n(\%)\end{array}$ & $p$ \\
\hline Género & & & 0.581 \\
\hline Hombre & 11 (19.3) & $5(20)$ & \\
\hline Mujer & 46 (80.7) & $20(80)$ & \\
\hline Edad & $38.8 \pm 14.2$ & $41.5 \pm 17.6$ & 0.506 \\
\hline Comorbilidades & & & 0.006 \\
\hline Sí & $10(17.5)$ & $12(48)$ & \\
\hline No & $47(82.5)$ & $13(52)$ & \\
\hline DM & $8(14)$ & $6(24)$ & 0.213 \\
\hline HAS & $5(8.8)$ & $5(20)$ & 0.114 \\
\hline $\mathrm{FA}$ & $0(0)$ & $1(4)$ & 0.305 \\
\hline Urolitiasis & $0(0)$ & $1(4)$ & 0.305 \\
\hline Hepatopatía & $1(1.8)$ & $2(8)$ & 0.219 \\
\hline Diagnóstico & & & 0.648 \\
\hline CCL & $39(68.4)$ & $18(72)$ & \\
\hline $\mathrm{CCL}+$ piocolecisto & $0(0)$ & $2(18)$ & \\
\hline $\mathrm{CCL}+$ hidrocolecisto & $9(14)$ & $3(12)$ & \\
\hline Quiste hepático & $0(0)$ & $1(4)$ & \\
\hline Absceso hepático & $0(0)$ & $1(4)$ & \\
\hline $\mathrm{CCL}+$ coledocolitiasis & $10(17.5)$ & $0(0)$ & \\
\hline Cirugía & & & 0.782 \\
\hline Colecistectomía laparoscópica & $57(100)$ & $22(88)$ & \\
\hline Drenaje laparoscópico & $0(0)$ & $2(8)$ & \\
\hline $\begin{array}{l}\text { Colecistectomía laparoscópica + colangiografía + } \\
\text { exploración de vías biliares }\end{array}$ & $0(0)$ & $1(4)$ & \\
\hline PIA inicio (mmHg) & $14 \pm 0.01$ & $13.9 \pm 0.4$ & 0.132 \\
\hline PIA durante la cirugía $(\mathrm{mmHg})$ & $14 \pm 0.0$ & $10.9 \pm 1.2$ & $<0.001$ \\
\hline PIA promedio $(\mathrm{mmHg})$ & $13 \pm 0.0$ & $12.6 \pm 0.7$ & 0.004 \\
\hline Dolor postquirúrgico & $6.2 \pm 1.92$ & $4.7 \pm 2.1$ & 0.002 \\
\hline
\end{tabular}


hacia el caudal para formar el ligamento hepatocólico. ${ }^{2}$

La irrigación de la vesícula biliar está provista por la arteria cística, que además irriga al conducto cístico. Habitualmente, esta arteria se origina de la arteria hepática derecha en un ángulo formado entre el conducto hepático común y el conducto cístico; a este espacio se le denomina triángulo de Calot o triángulo hepatocístico. Desde su origen, la arteria cística se dirige en sentido transversal hacia la derecha (o por atrás o por delante) del conducto hepático común. Esta arteria da dos ramas: una anterior y otra posterior, ambas rodean a la vesícula. ${ }^{1-3}$

Las venas císticas se encargan del drenaje venoso, tanto del cuello de la vesícula biliar como del conducto cístico. Estas venas entran directamente al hígado o drenan a la vena porta. En el caso del cuerpo y fondo, hay venas que pasan directamente a la cara visceral del hígado y drenan a los sinusoides hepáticos. ${ }^{3}$

El drenaje linfático se origina en las redes submucosas y se dirige hacia el ganglio cístico, que se ubica a nivel del cuello de la vesícula y hacia los ganglios de la raíz hepática. A partir de ahí, se dirige a drenar hacia los ganglios celíacos. ${ }^{4}$

La vesícula biliar y el conducto cístico están inervados por nervios que vienen en conjunto con la arteria cística desde el plexo celiaco, el cual provee las fibras simpáticas y viscerales aferentes para el dolor. Además, hay aporte del nervio vago para la regulación parasimpática y el nervio frénico derecho, que corresponde a las fibras somáticas aferentes. ${ }^{4}$

Habitualmente, la vesícula biliar posee una capacidad de almacenamiento de aproximadamente 30 a $60 \mathrm{~mL}$. Sin embargo, cuando está reabsorbiendo activamente agua, sodio, cloro y otros electrolitos de manera continua, pueden almacenarse hasta $450 \mathrm{~mL}$ de secreción. ${ }^{4}$

\section{Posición anormal de la vesícula biliar}

Las variaciones anatómicas en la vesícula biliar son comunes. No implican alguna patología, sin embargo, pueden dificultar la intervención quirúrgica, lo que predispone a que se cause una iatrogenia biliar. Por lo tanto, es importante tener en consideración esta posibilidad antes de desarrollar un procedimiento quirúrgico. ${ }^{1,3,4}$

Algunas de las variantes anatómicas más importantes son:

Vesícula biliar siniestra (vesícula situada a la izquierda del ligamento redondo): puede estar a la izquierda (cuando el hígado está en posición anatómica normal, es asintomática), intrahepática (debido a la aparición ectópica del esbozo), retrodesplazada, retroperitoneal, transversa o suprahepática. ${ }^{1-5}$

Vesícula biliar con gorro frigio: es una entidad clínicamente inocua consistente en un tabique (o un pliegue) parcial o completo, el cual separa el fondo y el cuerpo de la vesícula, debido a un defecto en la canalización a las doce semanas. Es de las variantes anatómicas más comunes. ${ }^{1,6}$

Vesícula biliar doble: el término de vesícula doble implica una duplicación de la misma en la que existen dos cavidades independientes y dos conductos císticos separados, que pueden desembocar por separado o reunirse previamente formando una "Y» invertida. Es una rara variante del sistema biliar con una frecuencia de uno por cada 4,000 casos, y puede ser detectada preoperatoriamente por estudios imagenológicos. La duplicación vesicular tiene una prevalencia de 2.5 en 10,000 estudios de autopsia, y aproximadamente 200 casos registrados en la literatura. Es importante resaltar que, en la mayoría de los casos reportados, el diagnóstico se realiza en estudios en cadáveres. ${ }^{1,7}$

Doble conducto cístico: la presencia de un conducto cístico se asocia hasta con $80 \%$ de los casos con una vesícula biliar doble; sin embargo, puede presentarse con una vesícula biliar única en menor cantidad de casos. La gran mayoría de las malformaciones del conducto cístico no tienen ningún significado clínico, tomando importancia al ser identificadas, ya sea con estudios de imagen o predominantemente de manera incidental durante un procedimiento quirúrgico. Es de vital importancia su iden- 
tificación para prevenir iatrogenia de esta estructura. ${ }^{1,8}$

\section{Colecistectomía laparoscópica}

La colecistectomía laparoscópica se realiza con el paciente en decúbito supino y con el cirujano ubicado a la izquierda del paciente y de frente al monitor. Existen varias técnicas de colecistectomía laparoscópica, entre las que destacan: la de incisión múltiple (MILS: Multi-Incision Laparoscopic Surgery), la de incisión única (SILS, por Single-Incision Laparoscopic Surgery) y la transluminal por orificio natural (NOTES por Natural Orifice Transluminal Surgery). ${ }^{1,15,16}$ A continuación, se desarrolla brevemente en qué consiste cada una de ellas.

MILS. Corresponde a la técnica clásica que se desarrolló a finales de 1980. El procedimiento se inicia con el establecimiento de un neumoperitoneo, el cual consiste en un espacio de trabajo que se crea insuflando un gas en la cavidad peritoneal. Existen dos técnicas para establecer el neumoperitoneo: una es la técnica cerrada, en la cual se accede "a ciegas» a la cavidad peritoneal; la otra es la técnica abierta, en la cual se accede a la cavidad peritoneal bajo visualización directa. En la técnica cerrada, se hace una incisión supraumbilical o infraumbilical en los tejidos abdominales superficiales y, a través de ella, se introduce una aguja de Veress. En la técnica abierta, se hace una incisión supraumbilical o infraumbilical y ésta se continúa en profundidad a través de la fascia y el peritoneo. Cuando se ha accedido a la cavidad peritoneal, se introduce en ella una cánula de Hasson y se hacen suturas de retención para anclar y asegurar la cánula a la fascia. Luego, se insufla el gas a través de la aguja de Veress o la cánula de Hasson y se crea el neumoperitoneo. Generalmente, el gas que se emplea es dióxido de carbono, aunque también se ha empleado óxido nítrico, argón y helio. ${ }^{16}$

Si se ha empleado la técnica cerrada, una vez que se ha establecido el neumoperitoneo, se introduce un trócar de 10 milímetros $(\mathrm{mm})$ por la incisión realizada y éste corresponde al puer- to o acceso umbilical. Si se ha empleado la técnica abierta, la cánula de Hasson corresponde al puerto o acceso umbilical. A través del puerto umbilical, se introduce el laparoscopio y se inspecciona el abdomen, en especial, la región de la vesícula biliar. Luego, se introduce un trócar de $10 \mathrm{~mm}$ en el epigastrio, un trócar de $5 \mathrm{~mm}$ en el hipocondrio derecho, aproximadamente en la línea medioclavicular derecha, y un trócar de 5 $\mathrm{mm}$ en el flanco derecho, aproximadamente en la línea axilar anterior derecha. ${ }^{16} \mathrm{~A}$ través de los trócares se inserta el instrumental quirúrgico, se realiza la resección de la vesícula biliar y finalmente ésta se extrae a través del puerto umbilical o del puerto del epigastrio. ${ }^{17}$

SILS. Las últimas dos décadas han significado una revolución en el campo de la cirugía mínimamente invasiva. Como parte de esta revolución, a finales de 1990 se desarrolló una técnica de colecistectomía laparoscópica, la cual se realiza con una única incisión umbilical y se denomina SILS.

En este caso, en la misma incisión umbilical se hacen diferentes incisiones en la fascia y, a través de ellas, se insertan los trócares. El principal objetivo del remplazo de la técnica MILS por la SILS es minimizar el carácter invasivo del procedimiento, con lo cual, teóricamente, se minimizan el dolor postoperatorio, la duración de la estadía hospitalaria y el tiempo de recuperación, al mismo tiempo que se optimiza la estética. No obstante, suele asociarse con un mayor tiempo operatorio. Se ha demostrado que es una técnica segura y una alternativa aceptable a la técnica MILS, y los estudios sugieren que reduce la estadía hospitalaria y el tiempo de recuperación en comparación con técnica MILS. ${ }^{17}$

NOTES. Como parte de la revolución en el campo de la cirugía mínimamente invasiva, también se ha desarrollado un nuevo enfoque en la cirugía endoscópica endoluminal. Esta cirugía se realiza introduciendo un endoscopio a través de un orificio externo, como la boca, el ano, la vagina o la uretra, para acceder a las diferentes cavidades corporales, y se denomina NOTES. 
La utilización de la técnica NOTES también tiene como objetivo minimizar el carácter invasivo del procedimiento. Entre las ventajas por las que destaca este procedimiento se encuentran: la reducción del dolor postoperatorio, la disminución de la analgesia requerida para la operación y la disminución del tiempo de recuperación. Además, al evitar incisiones superficiales, se evitan las cicatrices visibles, con lo cual se optimiza aún más la estética. ${ }^{17}$

La primera colecistectomía humana utilizando la técnica NOTES a través del abordaje transvaginal se reportó en 2007, y a través del abordaje transgástrico en 2008. El abordaje transvaginal se considera el abordaje de preferencia, porque elimina el riesgo de fuga de contenido intestinal y porque diversos registros han demostrado una asociación entre este abordaje y una baja tasa de complicaciones. Por último, el abordaje transcolónico para acceder a la cavidad peritoneal ha sido poco explorado, debido a la posibilidad de contaminación fecal y complicaciones infecciosas intraabdominales; sin embargo, sí se han reportado casos de colecistectomía siguiendo esta técnica. ${ }^{18}$

\section{Efectos fisiológicos de la cirugía laparoscópica}

La magnitud de los efectos cardiovasculares asociados con la laparoscopia va a depender de la interacción de varios factores, los cuales incluyen la posición del paciente a las presiones intraabdominales obtenidas durante la creación del neumoperitoneo, los efectos neurohumorales de la absorción de $\mathrm{CO}_{2}$, el estado cardiovascular previo, el volumen intravascular, la técnica de ventilación y los agentes anestésicos empleados. ${ }^{17}$

La mayoría de los estudios describen un aumento de la presión arterial media, resistencias vasculares sistémicas y presiones de llenado cardiaco, las cuales se pueden ver acompañadas de una disminución del índice cardiaco y cambios en la frecuencia cardiaca. ${ }^{16,18}$

La hipercapnia intencionada bajo anestesia aumenta el gasto cardiaco, la tensión arterial media, la frecuencia cardiaca y las concentraciones plasmáticas de catecolaminas. Las resistencias vasculares sistémicas disminuyen y reflejan los efectos vasodilatadores directos de $\mathrm{CO}_{2}$, cuando éstos no son contrarrestados por la activación del sistema nervioso simpático que constriñe los vasos de capacitancia. ${ }^{18,19}$

En todo procedimiento laparoscópico, se realiza insuflación de la cavidad peritoneal con gas. El $\mathrm{CO}_{2}$ es el gas que se utiliza con más frecuencia por su alta difusión, rápida eliminación y bajo costo. El capnoperitoneo separa la pared abdominal de los órganos para permitir el acceso visual. Los insufladores modernos liberan el gas con flujos de dos a $10 \mathrm{~L} / \mathrm{min}$. Se considera que una presión intraabdominal (PIA) de hasta $15 \mathrm{mmHg}$ es adecuada para la mayoría de los procedimientos quirúrgicos de abdomen superior. Con esta presión intraabdominal, en pacientes jóvenes y sin enfermedad concomitante, la retención de $\mathrm{CO}_{2}$ es mínima. Sin embargo, en sujetos con enfermedad cardiopulmonar, durante el capnoperitoneo, la absorción es mayor y los efectos hemodinámicos y pulmonares del $\mathrm{CO}_{2}$ son más significativos. ${ }^{19}$

El capnoperitoneo, la presión intraabdominal elevada, la hipercapnia y los cambios en la posición durante la cirugía laparoscópica inducen efectos fisiopatológicos a nivel cardiopulmonar, renal, hepático, cerebral y metabólico; éstos pueden complicar el manejo anestésico. ${ }^{18,19}$

Existen estudios de los cambios hemodinámicos en pacientes clasificados como ASA I. Lackey y colaboradores reportan resultados durante colecistectomía laparoscópica con el uso de monitoreo invasivo mediante catéter arterial pulmonar y a una presión intraabdominal de $14 \mathrm{mmHg}$. Por una parte, la inducción de la anestesia disminuye la presión arterial media (PAM) y el índice cardiaco (IC). El cambio de posición a Trendelenburg invertido reduce la presión de la aurícula derecha $(\mathrm{PAD})$, la presión capilar pulmonar en cuña (PCPC) y la precarga. La insuflación peritoneal incrementa la presión arterial media, la resistencia vascular sistémica (RVS) y la pulmonar (RVP), la presión de la aurícula derecha y la presión capilar pulmonar 
en cuña, sin cambios en la frecuencia cardiaca (FC). La combinación de los efectos de la anestesia, insuflación y posición producen una disminución en $50 \%$ del índice cardiaco. ${ }^{18}$

Por otra parte, el neumoperitoneo, al provocar un aumento de la presión intraabdominal, influirá sobre la cavidad torácica por elevación de los diafragmas, lo que, a su vez, ocasionará desórdenes fisiológicos, tales como: disminución de la compliance pulmonar, disminución de la capacidad funcional residual, aumento de la presión arterial de $\mathrm{CO}_{2}\left(\mathrm{PaCO}_{2}\right)$ y aumento del $\mathrm{CO}_{2}$ alveolar. ${ }^{19}$

Willis y su grupo de investigación estudiaron los cambios pulmonares y riesgos de complicaciones perioperatorias durante colecistectomía laparoscópica con capnoperitoneo a una presión intraabdominal de 10 a $15 \mathrm{mmHg}$ en pacientes con clasificación de ASA I. Durante la insuflación, obtuvieron cambios de los parámetros pulmonares, que incluyeron volumenminuto (VM), presión inspiratoria pico (PIP) y fracción espiratoria final de $\mathrm{CO}_{2}\left(\mathrm{FEFCO}_{2}\right)$. En pacientes con enfermedad pulmonar y/o cardiovascular, se impide el adecuado intercambio de gas con alteración en la absorción de $\mathrm{CO}_{2} \mathrm{y}$ suele ocurrir hipercapnia. La $\mathrm{PaCO}_{2}$ se eleva significativamente más que la $\mathrm{FEFCO}_{2}$, lo que indica aumento del espacio muerto pulmonar. Para mantener la $\mathrm{PaCO}_{2}$ entre $3,042 \mathrm{mmHg}$, se ajustan continuamente la frecuencia respiratoria $(\mathrm{FR})$, el volumen de cierre (VC) y se disminuye la presión intraabdominal. ${ }^{17,18}$

La manipulación del peritoneo parietal y de las vísceras abdominales, luego del neumoperitoneo, puede producir una estimulación vagal, lo que desencadenará los reflejos de náuseas, vómitos y la presencia de bradicardia. El vómito es la complicación más común, especialmente en pacientes obesos. La ansiedad en el preoperatorio, la distensión gástrica, la administración de opioides, el óxido nitroso, los anestésicos inhalatorios y el agente de reversión (neostigmina) pueden estar involucrados en la aparición de náuseas y vómito, que agravan el efecto facilitador de emesis que produce el neumoperitoneo y la posición de Trendelenburg. ${ }^{20}$
Una de las ventajas de la cirugía laparoscópica es la disminución del dolor, el cual suele presentarse luego de este tipo de procedimiento, y posterior a la cirugía, el $\mathrm{CO}_{2}$ tiende a acumularse en los espacios subdiafragmáticos e irrita el nervio frénico. Éste, a causa de metámeras, provocará un dolor a nivel de los hombros y la espalda (del cual se quejan los pacientes). Este dolor suele calmarse espontáneamente luego de varias horas mientras se absorbe el $\mathrm{CO}_{2}$; $\sin$ embargo, para aliviar la queja del paciente, se han empleado analgésicos no esteroideos como el ketoprofeno, el ketorolaco, entre otros. Una técnica preconizada por algunos anestesiólogos es la de dar oxígeno 100\% media hora después de haberse retirado el neumoperitoneo, para así estar seguros de que no quede gas carbónico en la cavidad peritoneal. ${ }^{18}$

Wahba y otros plantean que el dolor es de menor intensidad y más corta duración que el de una laparotomía. Éste ocurre esencialmente en el abdomen, la espalda y los hombros. La frecuencia de su presentación oscila entre 35 y $63 \%$ de pacientes y puede durar hasta tres días. ${ }^{17-20}$

El dolor en una sensación subjetiva, y su medición y análisis resultan difíciles. La escala visual análoga (EVA) del dolor, presentada como una barra horizontal de $10 \mathrm{~cm}$, es simple, factible y válida, ya que refleja el grado de intensidad del dolor en el momento de su evaluación, lo que la convierte en una herramienta útil para describirlo. Comparada con otras escalas, se piensa que es más sensible para la detección de pequeñas diferencias en los grados de dolor. ${ }^{16}$

El dolor escapular postquirúrgico, prácticamente inherente o propio a la cirugía laparoscópica, es un fenómeno frecuente (30-50\%) y molesto. Su etiología y patogénesis tampoco se han dilucidado del todo, por lo que existen múltiples teorías para tratar de explicar su origen. Hasta la fecha, los mecanismos propuestos incluyen: a) la sobredistensión diafragmática y de la cavidad abdominal, b) la irritación de las terminaciones diafragmáticas del nervio frénico, debida al ácido carbónico formado a partir del $\mathrm{CO}_{2}$ insuflado (esta teoría está apoyada por el hecho de que el dolor escapular disminuye si, en lugar 
de $\mathrm{CO}_{2}$, se insufla óxido nitroso), c) la activación del sistema nervioso simpático derivada de la hipercarbia que conduce a la amplificación de la respuesta inflamatoria tisular local, así como a la isquemia de la mucosa esplácnica y d) la irritación mecánica impuesta por los drenajes. ${ }^{18}$

Algunos estudios han mostrado ventajas potenciales con el uso rutinario de presiones bajas para mantener el neumoperitoneo en la práctica de la colecistectomía laparoscópica. Hace poco tiempo, la Asociación Europea de Cirugía Endoscópica publicó sus guías prácticas sobre neumoperitoneo para cirugía laparoscópica, dentro de las cuales recomienda usar la menor presión intraabdominal posible que permita una adecuada exposición del campo quirúrgico y no utilizar una presión rutinaria (recomendación grado B). De acuerdo con estas guías, una presión intraabdominal inferior a $14 \mathrm{mmHg}$ se considera segura en un paciente sano (recomendación grado A) y los dispositivos de elevación de la pared no muestran ventajas clínicas relevantes comparados con el neumoperitoneo de baja presión. ${ }^{18}$

No existe un acuerdo general con respecto a la presión a la que debe mantenerse el neumoperitoneo. Al respecto, tres estudios prospectivos y aleatorios ya publicados mostraron de manera significativa menor dolor postquirúrgico y escapular con tasas de conversión y complicaciones similares cuando la colecistectomía laparoscópica se practica con neumoperitoneo de baja presión. Por otro lado, algunos estudios más recientes que los anteriores no encontraron diferencias significativas en cuanto al dolor postquirúrgico entre pacientes sometidos a neumoperitoneo de baja presión y presión convencional, por lo cual se abstienen de recomendar el uso de neumoperitoneo con baja presión intraabdominal de manera rutinaria en la colecistectomía laparoscópica. ${ }^{19}$

Nuestro trabajo incluyó 82 individuos sometidos a colecistectomía por colelitiasis sintomática, siendo la mayoría mujeres. Del total, 57 fueron tratados de manera estándar con neumoperitoneo promedio de $14 \mathrm{mmHg}$ y 25 fueron manejados con neumoperitoneo con baja presión. Se encontró que sí hubo diferencia significativa en cuanto al dolor postquirúrgico en beneficio del neumoperitoneo de baja presión ( $p$ $=0.002$ ), lo cual es equiparable a lo reportado en la literatura médica.

\section{CONCLUSIONES}

No existieron diferencias en el resultado de cirugía de urgencia y programada con neumoperitoneo de baja presión.

No hubo diferencia significativa en la presión intraabdominal de inicio en ambos grupos.

Hubo disminución del dolor postoperatorio en colecistectomías laparoscópicas de neumoperitoneo de baja presión.

No se reportó ningún incremento de complicaciones postquirúrgicas secundarias a neumoperitoneo de baja presión comparado con neumoperitoneo estándar.

La colecistectomía con neumoperitoneo de baja presión tiene un impacto positivo en la recuperación postoperatoria de los pacientes.

Es factible la realización de este tipo de cirugías con neumoperitoneo de baja presión, siempre que se realice por un cirujano experimentado. De esta forma, también se mejoran los tiempos quirúrgicos y se favorece el alta precoz.

\section{BIBLIOGRAFÍA}

1. Nematihonar B, Fahimihanzaei H, Kamranmanesh M, Memary E, Shahbazi A, Mirkheshti A. Comparison postoperative shoulder pain, nausea, and vomiting between low and normal pressure pneumoperitoneum in laparoscopic cholecystectomy. Annals of Anesthesiology and Critical Care. 2017; 2 (2): 149-151.

2. Gurusamy KS, Koti R, Davidson BR. Abdominal lift for laparoscopic cholecystectomy. Cochrane Database Syst Rev. 2013; (8): CD006574.
3. Strasberg SM, Brunt LM. Rationale and use of the critical view of safety in laparoscopic cholecystectomy. J Am Coll Surg. 2010; 211 (1): 132-138.

4. Koc M, Ertan T, Tez M et al. Randomized, prospective comparison of postoperative pain in low- versus highpressure pneumoperitoneum. ANZ J Surg. 2005; 75 (8): 693-696.

5. Nasajiyan N, Javaherfourosh F, Ghomeishi A, Akhondzadeh R, Pazyar F, Hamoonpou N. Comparison 
of low and standard pressure gas injection at abdominal cavity on postoperative nausea and vomiting in laparoscopic cholecystectomy. Pak J Med Sci. 2014; 30 (5): 1083-1087.

6. Kar M, Kar JK, Debnath B. Experience of laparoscopic cholecystectomy under spinal anesthesia with lowpressure pneumoperitoneum: prospective study of 300 cases. Saudi J Gastroenterol. 2011; 17 (3): 203-207.

7. Vijayaraghavan N, Sistla SC, Kundra P et al. Comparison of standard-pressure and low-pressure pneumoperitoneum in laparoscopic cholecystectomy: a double blinded randomized controlled study. Surg Laparosc Endosc Percutan Tech. 2014; 24 (2): 127-133.

8. Schirmer BD, Edge SB, Dix J, Hyser MJ, Hanks JB, Jones RS. Laparoscopic cholecystectomy. Treatment of choice for symptomatic cholelithiasis. Ann Surg. 1991; 213 (6): 665-676.

9. Dexter SP, Vucevic M, Gibson J, McMahon MJ. Hemodynamic consequences of high- and low-pressure capnoperitoneum during laparoscopic cholecystectomy. Surg Endosc. 1999; 13 (4): 376-381.

10. Neudecker J, Sauerland S, Neugebauer E et al. The European Association for Endoscopic Surgery clinical practice guideline on the pneumoperitoneum for laparoscopic surgery. Surg Endosc. 2002; 16 (7): 1121-1143.

11. Barczyński M, Herman RM. A prospective randomized trial on comparison of low-pressure (LP) and standardpressure (SP) pneumoperitoneum for laparoscopic cholecystectomy. Surg Endosc. 2003; 17 (4): 533-538.

12. Serralta A, Bueno J, Sanhauja A, García R, Arnal C. Evolución del dolor postoperatorio en la colecistectomía laparoscópica bajo anestesia-analgesia multimodal en régimen ambulatorio. Rev Esp Anestesiol Reanim. 2002; 49: 461-467.
13. Dávila FSA, Chávez CRH. Dolor postoperatorio en colecistectomía laparoscópica. Ropivacaína vs placebo. Ensayo clínico. Cir Gen. 2010; 32 (2): 96-99.

14. Lucena JR. La irrigación con bupivacaína intraoperatoria en la prevención del dolor en el hombro post colecistectomía laparoscópica. Revista Multidisciplinaria del Consejo de Investigación de la Universidad de Oriente. 2005; 17 (2): 131-138.

15. Claros N, Fuentes I, Cari A, Laguna R. Omalgia asociada a neumoperitoneo a baja presión vs presión estándar durante la colecistectomía laparoscópica. Rev Med La Paz. 2010; 16 (2): 5-11.

16. López-Maya L, Lina-Manjarrez F, Díaz-Peralta A et al. Disminución del dolor postoperatorio en colecistectomía laparoscópica. Ropivacaína cutánea e intraperitoneal (antes vs después) de la incisión y neumoperitoneo. Rev Mex Anest. 2011; 34 (4): 251-259.

17. Schietroma M, Carlei F, Cecilia EM et al. A prospective randomized study of systemic inflammation and immune response after laparoscopic Nissen fundoplication performed with standard and low-pressure pneumoperitoneum. Surg Laparosc Endosc Percutan Tech. 2013; 23 (2): 189-196.

18. Basgul E, Bahadir B, Celiker V, Karagoz AH, Hamaloglu E, Aypar U. Effects of low and high intraabdominal pressure on immune response in laparoscopic cholecystectomy. Saudi Med J. 2004; 25 (12): 1888-1891.

19. Hua J, Gong J, Yao L, Zhou B, Song Z. Low-pressure versus standard-pressure pneumoperitoneum for laparoscopic cholecystectomy: a systematic review and meta-analysis. Am J Surg. 2014; 208: 143-50.

20. Donatsky AM, Bjerrum F, Gögenur I. Surgical techniques to minimize shoulder pain after laparoscopic cholecystectomy. A systematic review. Surg Endosc. 2013; 27: 2275-2282. 